\title{
An Ontology Based Remote Patient Monitoring Framework for Nigerian Healthcare System
}

\author{
Iroju Olaronke \\ Department of Computer Science, Adeyemi College of Education, Ondo, Nigeria \\ Email: irojuolaronke@gmail.com \\ Ojerinde Oluwaseun \\ Department of Computer Science, Federal University of Technology, Minna, Nigeria \\ Email: o.ojerinde@futminna.edu.ng
}

\begin{abstract}
There is a rapid increase in the demand of healthcare resources in Nigeria mainly healthcare personnel and healthcare facilities. This is due to the prevalence of chronic diseases such as endemic malaria, arthritis, hypertension, diabetes; an upsurge in the rate of avoidable deaths as well as an exponential increase in the population. However, healthcare resources in Nigeria are insufficient. For instance, in recent times, the ratio of doctors to the inhabitants is 1: 4,857 . Moreover, more than $60 \%$ of the Nigerian populace resides in rural areas where there are extreme shortages of healthcare practitioners and healthcare facilities due to geographical isolation and lack of opportunities. Hence, the low ratio of healthcare practitioners to patients causes a heavy workload on the healthcare practitioners. This however leads to medical errors as healthcare providers work under intense pressure to attend to the medical needs of their patients. This in turns leads to considerable loss of lives. In order to ameliorate this situation, this paper proposes an ontology based framework that will enable healthcare providers in Nigeria to continuously monitor their patients' health remotely outside the settings of the hospital. This will reduce the workload of the healthcare providers, assist them in decision making process as well as reduce the long waiting hours of the patients within the hospital environment. This framework is also designed to tackle the challenge of semantic interoperability facing healthcare systems around the globe.
\end{abstract}

Index Terms - Ontology, Remote Patient Monitoring, Semantic Interoperability, Healthcare System.

\section{INTRODUCTION}

Nigeria which is officially known as the Federal Republic of Nigeria is located on the western coast of Africa. Nigeria is the largest and the most populous country in Africa as well as the $7^{\text {th }}$ most populous country in the World, with an estimated population of over 177 million. Nigeria is currently undergoing population explosion and in line with this, the United Nations asserts that Nigeria will be one of the countries that will be responsible for the increase in the World total population by 2050 [1]. Nonetheless, Nigerians are prone to chronic diseases and malnutrition which are majorly caused by poverty [2]. Furthermore, most Nigerians are faced with enormous health challenges which are usually caused by incessant ethnic uproars, religious crisis as well as security crisis. In spite of these challenges, there are only 35,000 doctors in Nigeria which corresponds to 1 doctor per 4,857 inhabitants. This is farfetched from best practices and the global standard of 1 doctor per 600 inhabitants of a country [3]. The major reasons for the inadequate number of physicians can be attributed to brain drain as well as the refusal of most doctors to serve in rural areas where about $60 \%$ of the total population resides [4]. Consequently, the Nigerian populace especially those who reside in rural areas have limited access to basic healthcare services which has resulted in the persistence of high disease burden. In addition, medical practitioners work under intense pressure due to the heavy workload caused by the large percentage of patients they attend to on a daily basis. In line with this, Varshney [5] pointed out there is a potential link between an increase in medical errors and cognitive overload of medical practitioners. Hence, the Nigerian healthcare system is saddled with a high rate of errors which results in a high rate of mortality. In order to ameliorate this problem, this paper is of the view that patients' health be continuously monitored by healthcare practitioners remotely outside the settings of the hospital. This is with the aim of reducing the number of patients that medical practitioners attend to on a daily basis. Hence, this paper presents an ontology based framework for remote patient monitoring in Nigerian healthcare system so that the populace can have access to basic healthcare services regardless of their geographical locations. The framework is designed to be more convenient than traditional care and it also tackles the challenge of semantic interoperability facing the healthcare industry. This is with a view to assisting the health system to be more vibrant as no country can have a steady economic growth in the absence of an adequate healthcare system.

The remaining part of this paper is organized as follows: sections 2 and 3 deal with the overview and advantages of remote patient monitoring; section 4 deals with existing remote patient monitoring systems; the proposed framework and models are designed in sections 
5 and 6 , the distinctive feature of the proposed model is highlighted in section 7 . The challenges of the model in the Nigerian context are discussed in section 8 ; section 9 concludes the study.

\section{OVERVIEW OF REMOTE PATIENT MONITORING}

Patient monitoring according to Hudson et al. can be viewed as the regulated observation or measurement of a patient's physiological function and the function of life support equipment; which is done with the aim of building management decisions, as well as making therapeutic interventions and assessing those interventions [6]. The Continua Health Alliance [7] defines remote patient monitoring (RPM) as a technology that enables the monitoring, evaluation and management of an individual through a remote interface that collects clinical data from the individual and then transmits the data to a remote healthcare provider for clinical review, care management, and patient education. Remote patient monitoring also refers to a wide range of technologies which enables patients' vital signs and other physiological functions to be monitored, evaluated and managed remotely especially outside the conventional settings of the hospital such as the home. Patients' physiological functions usually monitored include heart rate, oxygen saturation, glucose levels, fetal movement in pregnant women, blood pressure, respiratory rate, and body temperature.

The process of monitoring a patient remotely includes five basic steps. These include the collection of patients' information, transmission of the information, information evaluation, notification and intervention [8]. The collection phase involves the active or passive gathering of patients' physiological data through sensor based peripheral devices such as blood pressure cuff, pulse oximeter and glucometer which are usually worn by the patient. The data collected are then transmitted to healthcare practitioners through different modes of communication where they are reviewed and evaluated by algorithms or healthcare providers who provide clinical review, care management and patient education. In cases of emergencies, the patient is notified through a mobile device or an intermediary healthcare provider who takes the necessary intervention. The remote patient monitoring process is illustrated in Fig. 1.

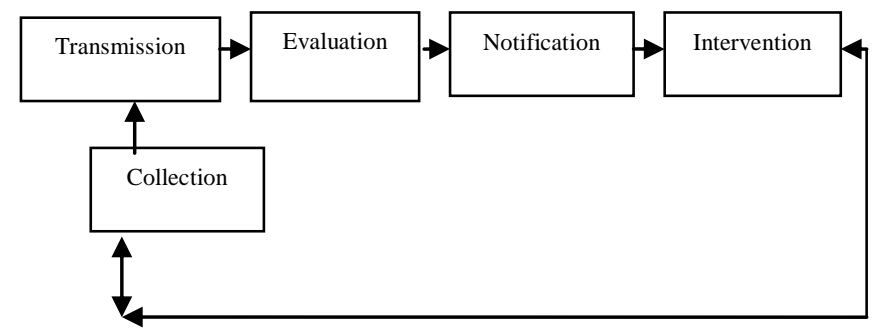

Fig.1. Processes involved in remote patient monitoring [8]

Basically, Smith and Sweeney [9] admit that most remote patient monitoring technologies follow a general framework that consists of four basic components. These components include sensors on the peripheral devices, local data storage, centralized data repository as well as diagnostic application software. The sensor based devices can be wearable, implantable or installed in the home. A wireless Body Area Network (BAN) allows the patient's data to be collected by the sensors. The local data storage is usually located at the patients' sites to store the data measured. The patients' data are transmitted via wireless or wired communication techniques to the centralized data repository where authorized medical staff can access the data. The diagnostic application software analyzes the data and develops treatment plans and provides intervention alerts based on the data analyzed.

Lui [10] is however of the view that the framework of a remote patient monitoring system consists of three major components. These include data acquisition, data transmission as well as the central control room. In this framework, the vital signs of a patient are measured via sensor enabled devices and acquired by a bedside monitor and then transmitted through a network to the central control room which is responsible for monitoring, analyzing and storing patients' data.

\section{AdVAntages of Remote Patient Monitoring}

The healthcare system is generally under intense pressure as the access to timely information is a major challenge. In addition, the few healthcare practitioners that attend to a large number of people with chronic diseases pose a challenge to the healthcare system. Hence, the need to remotely monitor a patient's health outside the conventional hospital environment becomes pertinent.

Remote patient monitoring has long been beneficial to both patients and healthcare providers. One of the major benefits of remote patient monitoring is that it enables healthcare practitioners to have consistent and timely information about their patients which ultimately allow healthcare providers to proactively and efficiently manage their patients' health through best practices. This section thereby provides more benefits of remote patient monitoring.

\section{A. Reduction in the Cost of Care}

One of the major challenges that the healthcare industry is facing is increasing costs [11]. For instance, the cost of healthcare in the United States of America was about $16.9 \%$ of the Gross Domestic Product in 2012 [12] 
However, the Center for Technology and Aging asserts that the use of remote patient monitoring systems would reduce the cost of healthcare by nearly $\$ 200$ billion in the next 25 years [8]. Hence, remote patient monitoring is more cost effective than traditional care.

\section{B. Increased Access to Healthcare}

Remote patient monitoring provides increased access to patients' care even in their homes. Hence, patients feel more confident and supported whenever there is a challenge in their health. This is because they are aware that their health is being constantly monitored by a professional healthcare provider. Furthermore, barriers such as those caused by distance are eliminated as patients especially those in rural areas where there are inadequate healthcare facilities do not need to travel long distances for healthcare services.

\section{Early Detection of Abnormalities}

Remote patient monitoring facilitates the early detection of abnormalities or deterioration in patients' health. This is because patients' health are constantly monitored. Hence, remote patient monitoring prevents serious health complications, emergencies and hospitalization. This increases the safety of the patient.

\section{Reduction in the Rate of Mortality}

Remote patient monitoring systems reduce mortality rate. For instance Smith et al. [13] emphasized that patients who suffer cardiac arrest and require unexpected admission to Intensive Care Unit usually show premonitory abnormalities in vital signs. Hence, the constant monitoring of such patients' vital signs will facilitate their recovery process and thus reduce the rate of mortality.

\section{E. Increased Participation of Patients' in their own Care}

Remote patients monitoring devices increase patients' participation in the management of their own health. Consequently, remote patient monitoring facilitates communication and trust amongst patients' and healthcare providers.

\section{EXISTING REMOte PATIENT MONITORING SySTEMS}

A lot of efforts have been made towards the design of remote patient monitoring systems. However, most of these systems are associated with a lot of challenges. Nonetheless, this section provides a review of existing remote patient monitoring systems.

Alexandra et al. developed a mobile phone based remote patient monitoring system for the management of hypertension in diabetic patients [14]. The system comprised a Bluetooth-enabled home blood pressure (BP) monitor and a mobile phone. The function of the mobile phone is to receive home readings which are transmitted wirelessly from the BP monitor to a server. The patients also receive blood pressure alerts on their mobile phones. The system is also composed of a central server for data processing, a fax-back system to send physicians' reports and a blood pressure alerting system which sends a signal to the physician if a patient's blood pressure exceeds a particular threshold. The system was designed to ensure the confidentiality of patients' data as it only transmits and receives data from predetermined mobile phones. However, patients with low dexterity or visual impairment found the use of the system very challenging and frustrating [14]

Lui developed a wireless remote patient monitoring system on general hospital wards which allows patients'vital signs to be gathered and sent to a control unit for centralized monitoring [10]. The system deployed the use of five sensors to measure a patient's physiological functions (heart rate, oxygen saturation, blood pressure, respiration rate and temperature). These sensors were networked through a wireless network technology specifically ZigBee. A master node which was integrated with the five sensors forms a Wireless Personal Area Network (WPAN). This controls the communication of the sensors by collating and sending the vital signs to a central control unit through an Ethernet-based Local Area Network. This system however is limited to hospital wards as the patients' health could not be monitored outside the conventional setting of the hospital.

The Ubiquitous Monitoring Environment for Wearable and Implantable Sensors (UbiMon), a project by Imperial College London, United Kingdom, provides a continuous and unobtrusive monitoring for patients with arrhythmic heart disease [10]. The system comprises a Bluetoothbased WPAN worn by a patient to collect vital signs. The vital signs are then transferred to a PDA and then processed by the PDA which is carried by the patient. The PDA sounds an alarm whenever an abnormality is discovered in the patients' vital signs. The patient then sends the message to his healthcare provider for diagnosis, treatment or intervention. Nevertheless, the major limitation of this system is that it requires manual operations by the patients.

MobiHealth developed the MobileHealth remote patient monitoring system which also monitors a patient's health outside the setting of the hospital [15]. MobiHealth is similar to UbiMon because it comprises a BluetoothWPAN and a PDA used the same way as in the Ubicon. However, MobiHealth automatically sends notification to medical personnel through General Packet Radio Service (GPRS)/Universal Mobile Telecommunication System (UTMS) message services, which minimized patient's manual operations. One of the major limitations of this system is that the number of sensors that can be used is restricted since a Bluetooth WPAN can only support up to seven active nodes for transmission in RPM [10]. In addition, this system does not support patient feedback. Furthermore the Bluetooth WPAN can only work for 9 hours to support continuous local monitoring [15].

Sebastian et al. also developed a remote patient monitoring system which captures the Electrocardiogram (ECG) signals of patients in an Intensive Care Unit (ICU) [16]. The system deployed a web camera which captures the images from the screen of a bedside monitor. These 
images are then processed in the matrix laboratory (MATLAB) environment and uploaded into an application server. Once an abnormality is discovered in the patient's vital signs, the doctor is notified via his mobile phone. The basic limitation of this system is that the notification is sent to all doctors who are authorized to use the system. Hence, this system does not consider patients' privacy and confidentiality.

Abayomi-Alli et al. designed and implemented a mobile remote health monitoring system on wireless technologies which is largely a mobile agent based system [17]. The system made use of three major networks which include the Body Area Network (BAN), the Personal Area Network (PAN) and the Wide Area Network (WAN). The BAN contained the sensors for monitoring patients' bio-signals. The PAN consists of the BAN and it connects the supervisor's agent and the mobile base unit (MBU) through Bluetooth technology. The WAN connects the PAN, mobile response unit (MRU) and hospital site (HS) through GPRS and Internet technology. The system employed the use of Hyper Text Transfer Protocol (HTTP) over Secure Socket Layer (SSL) and Transport Layer Security (TLS) protocol to secure data transmission during user authentication. This is done to ensure confidentiality and data integrity. The major limitation of the system is that it requires manual operation from the patients as it requires patients to send
Short Message (SMS) to paramedics or other medical staff.

\section{The Proposed Remote Patient Monitoring FRAMEWORK}

In this section, the framework of the proposed remote patient monitoring system is designed. The algorithm depicting the ontology mapping process involved in the system is also presented in this section. The ontology based remote patient monitoring framework is as shown in Fig. 2. The framework supports the use of Zigbee based sensor devices to measure a patient's vital signs rather than Bluetooth sensor based devices. This is because Zigbee technology is simpler, less expensive and more power efficient. Zigbee technology also has a longer transmission range and can accommodate more sensors than the Bluetooth technology [10].The sensor based devices are designed to be worn by the patients because they are non-invasive. The healthcare provider recommends the sensors to the patients based on their health challenges. The patients are allocated a unique patient identification number (PID) and the sensors are also allocated a unique device identification number. These identification numbers are assigned to each of the patient's sensors.

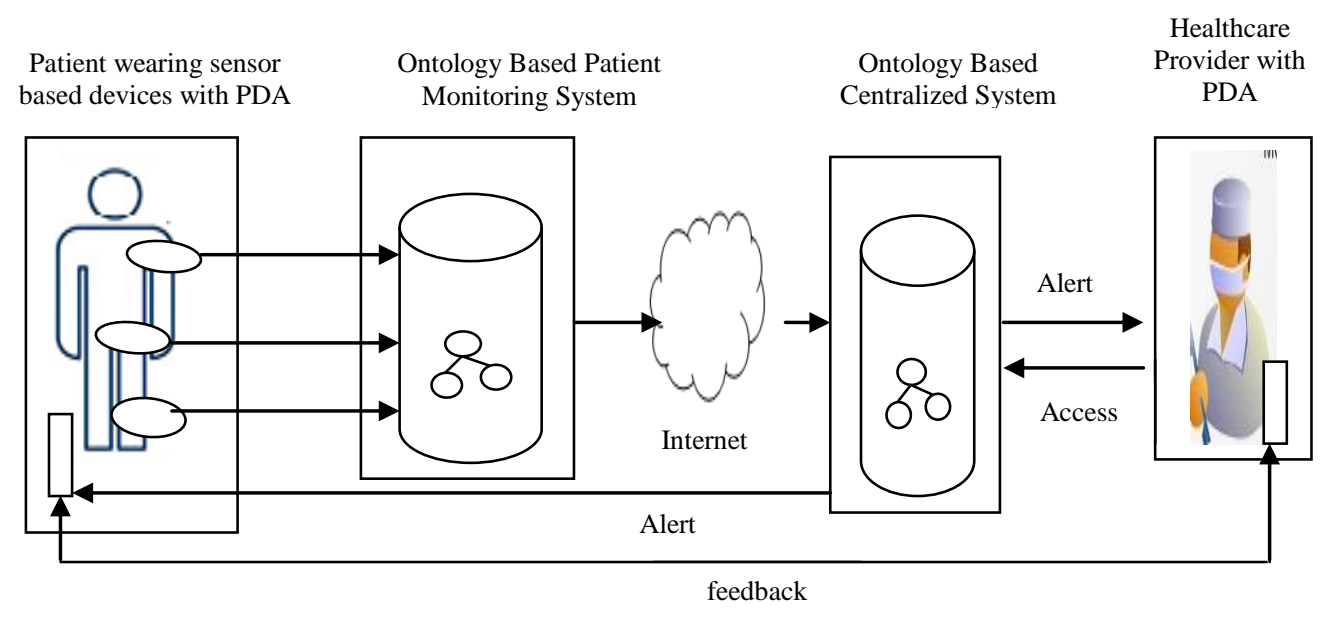

Fig.2. The remote patient monitoring framework

The function of the PID is to uniquely identify the patient whose vital signs are being measured while the unique device identification number facilitates the identification of the sensor transmitting the patient's data at a particular period. The sensors are only activated if the patients have been authenticated through their biometrics such as finger print authentication. This is to prevent a wrong user from wearing the sensors. The basic function of the sensor devices is to measure the patient's vital signs which are collected through the body area network. The vital signs are transmitted to an ontology based patient monitoring system which is located at the patient's site through a Zigbee network. The interconnection between the sensor based devices and the patient monitoring system forms a Wireless Personal
Area Network (WPAN). The function of the patient monitoring system is to collect, store and display the values of the patient's vital signs. Furthermore, the patient's information is transmitted to the ontology based centralized database located at the hospital site via the Internet. The Internet is used because it spans a wide geographical area. However, the Internet is prone to a lot of attacks. Hence, this system employs intrusion detection and prevention system, user authentication and user authorization to ensure security.

During information exchange, the intrusion detection and prevention system monitors the network for malicious activities. This is achieved by notifying the security administrators of the patient monitoring system through an alarm if a malicious activity is detected. The 
system blocks the network traffic from the suspected malicious source and then shuts down the network connection. However, if no intrusion is detected, the process of system authentication takes place. System authentication involves the use of login details which includes the user identification credentials and a secret password. This is to prevent a wrong user from having access to the system. The authorization system is a rule based system which is based on the roles of the users. Users are granted a particular view of the system based on the functions they perform. For instance, only a doctor that the patient is consulting is allowed to view the patient's vital signs. This process is to ensure confidentiality, reduce misuse of patients' information and control access to the system. The ontology based centralized system is also a rule based detection system which automatically alerts the patient and his healthcare provider through their Personal Digital Assistants (PDA) or mobile phones when the vital signs of the patients is outside the range of a particular threshold. At the point of emergency, the healthcare provider and the patient may communicate on further modes of treatment, health education and other proactive actions through their PDAs

Both the patient monitoring system and the centralized system in the framework are ontology based. This is because the use of ontologies provides ways of representing and organizing medical terminologies which facilitate seamless communication of patients' information. Furthermore, the use of ontologies according to Shvaiko [18] provides the possibility of sharing a common comprehension of the structure of the information exchanged. Hence, the use of ontology was proposed so that a shared vocabulary can be provided for the two systems to understand the information exchanged. However, since both systems are located at different sites, there is a possibility that the ontologies are proprietary and heterogeneous. Hence, semantic heterogeneity becomes a challenge at this point. It therefore becomes pertinent to reconcile the onologies by mapping them. Mapping the two heterogeneous ontologies compares the senses or semantics of a term by finding a term $T_{i}$ in the patient monitoring ontology that has the same or closest intended meaning with a term $T_{j}$ in the centralized ontology from a knowledge base resource such as the Unified Medical Language System (UMLS). The synonyms of both terms are also looked up in the methatherus of the UMLS. If a common term is found in the list of synonyms of both terms $\mathrm{T}_{\mathrm{i}}$ and $\mathrm{Tj}$, there is a possibility that both terms are synonymous. For instance, if the word heart rate is represented as cardiac rate in the ontology of the patient monitoring system and as heart rate in the ontology of the centralized system, the ontology mapping process will reconcile these two terms so that they can easily be understood by both communicating systems and users and treated as synonymous terms. This will resolve semantic heterogeneity in the proposed framework. The ontology mapping process is illustrated in algorithm 1.

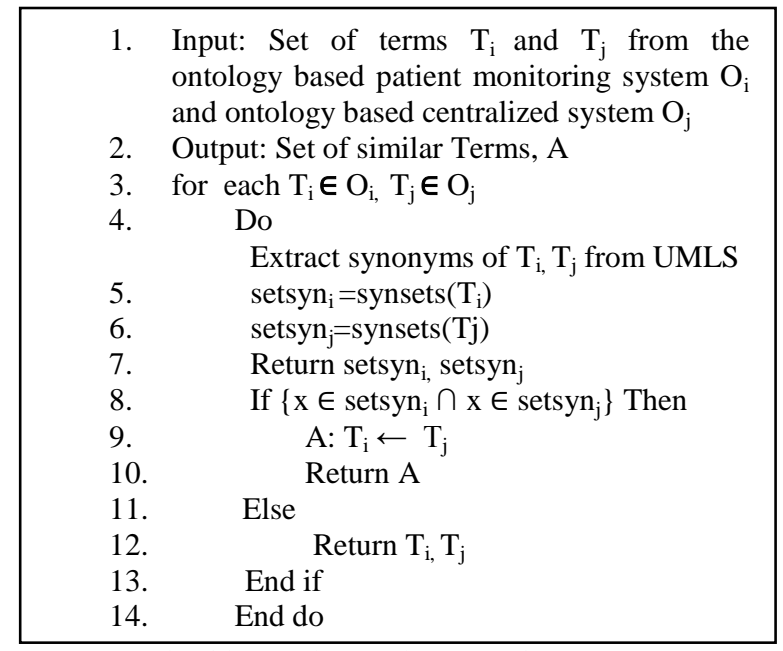

Algorithm 1. The Ontology Mapping Process

\section{THE MOdELS OF THE PROPOSED FRAMEWORK}

It is expedient that the requirements of the remote patient monitoring framework are identified. These requirements are emphasized using the use case and activity diagrams of the Unified Modeling Language (UML). The use case diagram is designed to graphically depict the interactions between the system and its users. It describes who use the system and in what way the user interacts with the system. The following components used for the design of the use case diagrams include actor and use case. The actor is represented as a stick figure. It lies outside the system model and interacts with it. The use case is represented as an oval shape. It describes a sequence of interactions between actors and the system. The use case diagram of the remote patient monitoring framework is as shown in Fig. 3. The activity diagram of the proposed framework is also shown in Fig. 4. The activity diagram captures the dynamic behavior of the system and models the use case at a more detailed level. It also shows the flow of messages from one activity to the other.

\section{The Distinctive FEATURE OF THE PROPOSED FRAMEWORK}

Healthcare terms are usually polysemous in nature and as a result it becomes difficult for computers and Information and Communication Technology (ICT) tools to capture the semantics of information exchanged amongst diverse systems. Hence, healthcare systems are usually associated with a high level of inconsistencies or discrepancies in the meaning and the interpretation of the same or related medical terms. Consequently, it becomes pertinent to introduce the concept of ontology mapping process into the remote patient monitoring framework. This is to ensure that the two systems exchanging information, that is, the patient monitoring system and the centralized system exchange information with a shared and unambiguous meaning. Hence, one of the major features that distinguish the proposed remote patient 
framework from existing systems is the ontology mapping process. Furthermore, this framework supports patients' feedback as it provides an opportunity for patients to communicate their health challenges to their healthcare providers once they receive an alert indicating an abnormality in their vital signs. In addition, the proposed framework is role based, hence, healthcare providers will only have access to patients' data based on the functions they perform. For instance, a doctor who is not monitoring a particular patient might not be allowed to view the patient's vital signs.

\section{Challenges of THE PRoposed Framework IN THE NIGERIAN CONTEXT}

The remote patient monitoring system is characterized by a lot of challenges in spite of its numerous benefits. The factors hindering the effective deployment of the proposed framework include:

\section{A. Erractic Power Supply}

Wearable sensors and computing devices involved in the remote patient monitoring system are usually powered by electrical power supply such as batteries. However, the electrical power supply of a battery is limited unless it is continuously charged. This might not be realistic in the Nigerian setting as a result of erratic power supply. Thus, it becomes difficult to continuously monitor the patients' health.

\section{B. Ethical Issues}

The healthcare industry is plagued with fraud and insecurity and as a result, ethical issues such as the protection of patients' data, the safety of the patient, patients' confidentiality and consent, data privacy, data integrity, control of access to patients' information can discourage the effective deployment of the remote patient monitoring system.

\section{Reluctance to Change}

The Nigerian health system is majorly paper based and as a result most healthcare providers have a low level of computer literacy. Consequently, the healthcare providers are usually unwilling to change from the traditional way of care to new technological developments.

\section{Trust}

Trust is a very vital component that is required for building effective patient-physician relationship. Hence, patients' would not be able to share their information with their healthcare providers if there is no trust between them. Hence, if there is no trust, the deployment of the remote patient monitoring system might be difficult as the patient might find it difficult to share their information which is a significant element in the remote patient monitoring system.

\section{E. Low Bandwidth}

The proposed remote patient monitoring system is Internet based. However, a larger percentage of the Nigerian populace who reside in the rural areas has low Internet bandwidth or no access to the Internet. This will definitely hinder the effective deployment of the remote patient monitoring model.

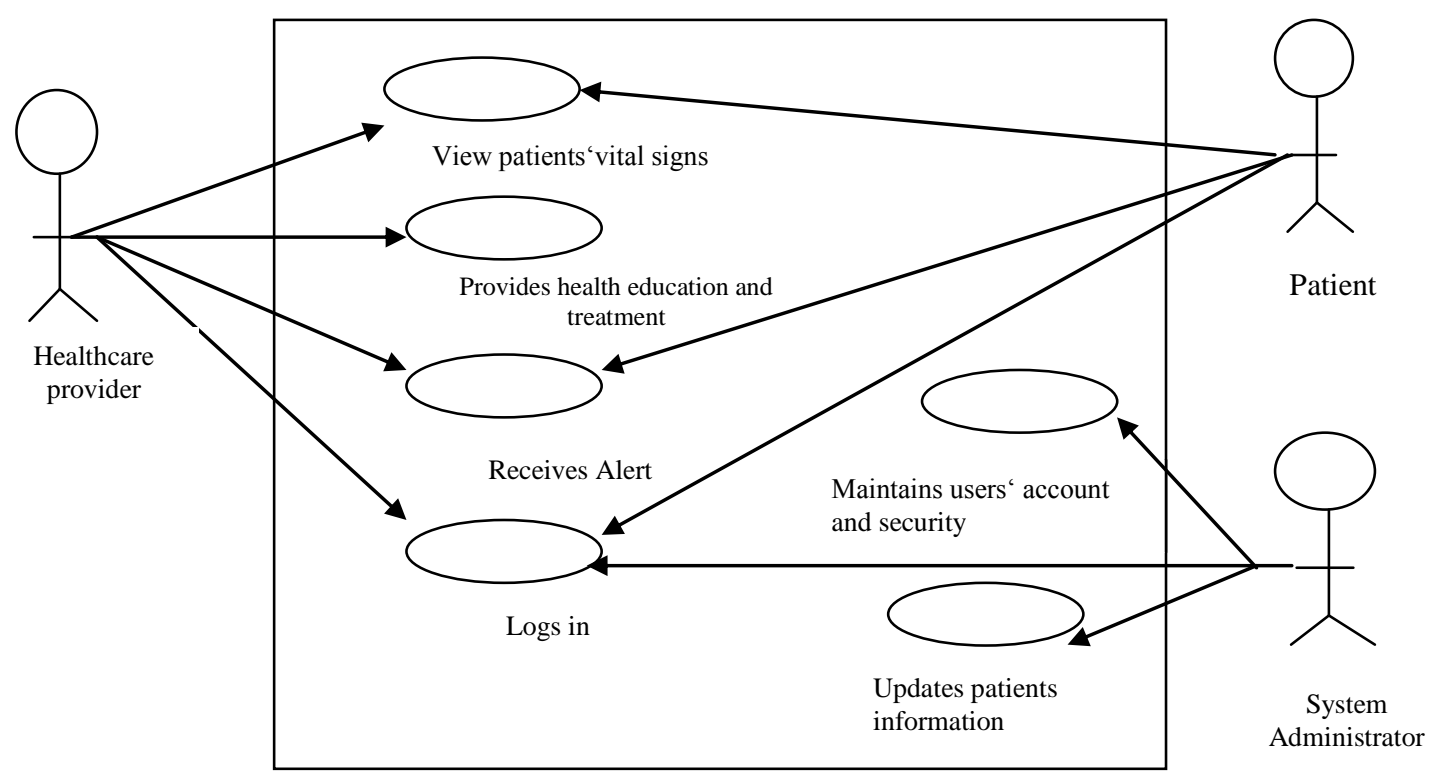

Fig.3. The use case diagram of the proposed remote patient monitoring framework 


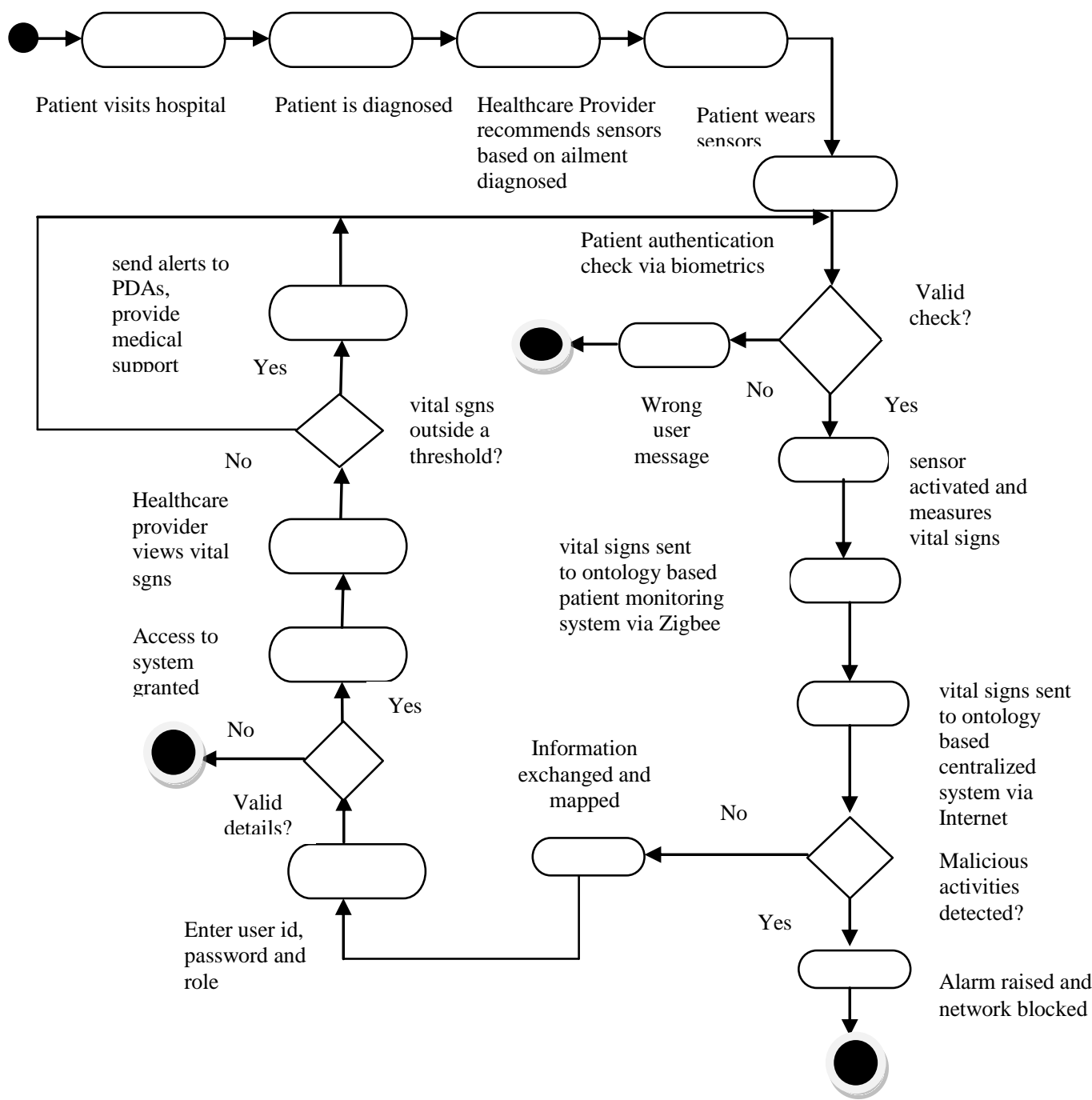

Fig.4. The activity diagram of the proposed remote patient monitoring framework

\section{CONCLUSION}

Remote patient monitoring system offers diverse technologies which enable patients' vital signs and other physiological functions to be monitored remotely especially outside the conventional settings of the hospital. The benefits of remote patient monitoring system include increased access to healthcare services, reduction in medical errors, early detection of abnormalities in patients' health as well as an increase in patients' participation in their own care. Consequently, a remote patient monitoring framework was proposed for the Nigerian healthcare system. This is majorly due to the exponential increase in the total population as well as the low healthcare provider to patients' ratio. The proposed framework is ontology based because of the problem of semantic heterogeneity facing healthcare systems around the globe. Furthermore, the concept of ontology mapping was introduced into the framework because ontologies can be heterogeneous which also poses a threat to semantic interoperability in healthcare. Hence, the ontology mapping process will enhance the semantic interpretation of the vital signs terms exchanged by the communicating systems in the proposed framework. Nonetheless, factors such as ethical issues, trust, low bandwidth and erratic power supply have been identified as some of the challenges that affect the effective implementation and deployment of the remote patient monitoring framework in the Nigerian setting.

\section{REFERENCES}

[1] United Nations Children Emergency Fund, The Nigerian Situation, United Nations Children Emergency Fund, 2007.

[2] C. Ucha, Poverty in Nigeria: Some Dimensions and Contributing Factors, Global Majority E-Journal, pp. 4656, 2010.

[3] J. Esangbedo, Paradox of Doctor Shortage in Nigeria, The Guardian Newspaper, November 30, 2015.

[4] R Akinyede, O. Daramola and O. Adewale, Towards Delivering High Quality Healthcare through an Integrated National Health Information System and Telemedicine Development, Problems And Recommended Solutions, Canadian Journal on Computing in Mathematics, Natural Sciences, Engineering and Medicine, pp. 180-187, 2012

[5] U. Varshney, "Enhancing Wireless Patient Monitoring by 
Integrating Stored and Live Patient Information", 19th IEEE International Symposium on Computer-Based Medical Systems, Salt Lake City, USA, pp. 501-506, 2006

[6] O.Akomolafe and O. Emuoyibofarhe, A Context Aware Mobile Agent Middleware Framework for Remote Patient Monitoring for LAUe-HCMS, Ladoke Akintola University of Technology Ogbomoso, Oyo State,Nigeria, 2013

[7] Continua Health Alliance, Remote Patient Monitoring, Continua Health Alliance, 2010.

[8] Center for Technology and Aging, Technologies for Remote Patient Monitoring for Older Adults, Center for Technology and Aging, USA, 2010

[9] T. Smith and R. Sweeney, Fusion Trends and Opportunities, Medical Devices and Communications, 2010

[10] Y. Lui, Wireless Remote Patient Monitoring on General Hospital Wards, Bournemouth University, 2010

[11] C. Bock, L. Carnahan, S. Fenves, M. Gruninger, V. Kashyap, B. Lide, J. Nell, R. Raman, and R. Sriram, Healthcare Strategic Focus Area: Clinical Informatics, National Institute of Standards and Technology, Technology Administration, Department of Commerce, United States of America, pp. 1-33, 2005

[12] OECD Health Statistics, How Does the United States Compare, http://www.oecd.org/health/healthdata, 2014

[13] G Smith, D. Prytherch, P. Schmidt, P. Featherstone, D. Knight, G. Clements, and M. Mohammed, Hospital-wide Physiological Surveillance-A New Approach to the Early Identification and Management of the Sick Patient, Resuscitation, pp. 19-28, 2006

[14] G. Alexander, J. Warren, A. McIsaac, I Jane, S. Allison, D. Andrea, A. Carlos, S. Denice, H. Melinda, T. Mathieu and A. Joseph, A Mobile Phone-Based Remote Patient Monitoring System for Management of Hypertension in Diabetic Patients, American Journal of Hypertension, 2007

[15] MobiHealth, MobiHealth http://www.mobihealth.org , 2008

[16] S. Sebastian, N. Jacob, Y. Manmadhan, V.R. Anand, and M. Jayashree, Remote Patient Monitoring System, International Journal of Distributed and Parallel Systems, pp. 99-110, 2012
[17] A. Abayomi-Alli, Ikuomola A., O. Aliyu, and O. Abayomi-Alli, Development of a Mobile Remote Health Monitoring system - MRHMS, African Journal of Computing and ICT, 14-22, 2014

[18] P. Shvaiko, Iterative Schema-Based Semantic Matching, International Doctorate School in Information and Communication Technology, University of Trento, 2006

\section{Authors' Profiles}

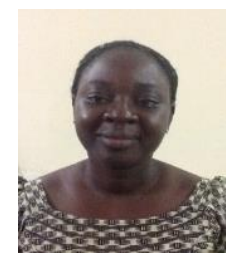

Dr. Iroju Olaronke has a B.Sc. in Computer Technology at Babcock University, Nigeria. She also has M.Sc and $\mathrm{PhD}$ in Computer Science at Obafemi Awolowo University, Nigeria. She is a lecturer at the Department of Computer Science, Adeyemi College of Education, Ondo, Nigeria. Her research interest is on health informatics, interoperability and ontology matching.

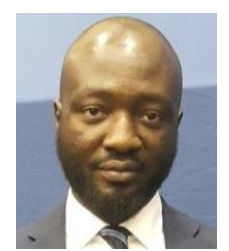

Dr. Oluwaseun A. Ojerinde is a lecturer in the Department of Computer Science in the School of Information and Computer Technology in Federal University of Technology, Minna. He bagged his B.Sc. in Computer Technology at Babcock University in 2006. He received his M.Sc. in Mobile Communication System from Loughborough University in 2008 $\mathrm{He}$ also obtained his $\mathrm{PhD}$ in Mobile Communication System from Loughborough University in 2014. His research area is in Antenna, On-body systems, Multiple Input Multiple Output (MIMO) systems, spanning, Telecommunications, Networking and Radiation. He has worked on the effects of metallic objects on radiation for mobile devices. He is a member of IEEE and IET.

How to cite this paper: Iroju Olaronke, Ojerinde Oluwaseun,"An Ontology Based Remote Patient Monitoring Framework for Nigerian Healthcare System", International Journal of Modern Education and Computer Science(IJMECS), Vol.8, No.10, pp.17-24, 2016.DOI: 10.5815/ijmecs.2016.10.03 